\title{
Regge amplitudes from AdS/CFT duality
}

\author{
R. Peschanski ${ }^{\text {a }}$

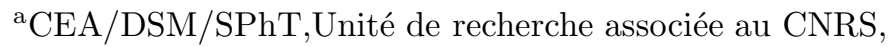 \\ CE-Saclay, F-91191 Gif-sur-Yvette Cedex,France; \\ email: pesch@spht.saclay.cea.fr
}

\begin{abstract}
String theory has long ago been initiated by the quest for a theoretical explanation of the observed high-energy "Regge behaviour" of strong interaction amplitudes, but this 35-years-old puzzle is still unsoved. We discuss how modern tools like the AdS/CFT correspondence give a new insight on the problem.
\end{abstract}

\section{Introduction}

It is well-known that string theory started from the proposal of scattering amplitudes which may grasp the two major structures of soft interaction phenomenology in a condensed form : resonances and Regge poles. Two types of amplitudes were proposed for four-point amplitudes, see Fig.1. The Veneziano amplitude corresponds to Reggeon exchanges with non-vacuum quantum numbers and the Shapiro-Virasoro amplitude corresponds to Pomeron exchange with vacuum quantum numbers. As soon after demonstrated, they correspond to respectively open and closed bosonic string theory amplitudes at treelevel.

However, despite many efforts during years, no widely recognized progress have been done in the string theory of strong interaction amplitudes, and after the discovery of QCD as the gauge field theory of quarks and gluons, there remained little place for it. Indeed, major theoretical obstacles have been raised, for instance:

- The conformal anomaly of string theories in Minkowski $D$-dimensional space leads to the limitation $D=26,10$ for bosonic and super strings.

- Zero mass gauge and gravitational fields appear in the string spectra of asymptotic states, if not tachyonic states.

To these (non exhaustive list of) difficulties, new questions have to be added after the discovery of QCD, for instance:
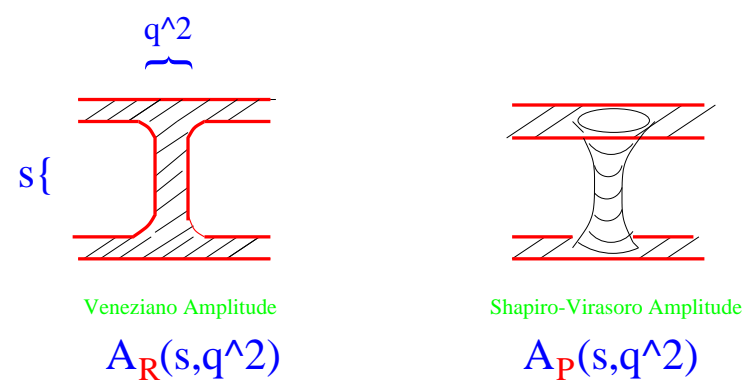

Figure 1. Reggeon and Pomeron String Amplitudes.

- Where are "hard" interactions recovered in a string theory framework?

- Can we elaborate a suitable string Theory which could coherently describe the properties of gauge fields?

To these pending questions, the recently proposed AdS/CFT correspondence between certain string and gauge field theories may give new and reliable answers. Among these questions, the understanding of Regge amplitudes in terms of the AdS/CFT duality have been the subject of an approach which I will now describe.

\section{String/Gauge fields Duality}

The AdS/CFT correspondence [1] has many interesting formal and physical facets. Concerning the aspects which are of interest for our problem, it allows one to find relations between gauge 


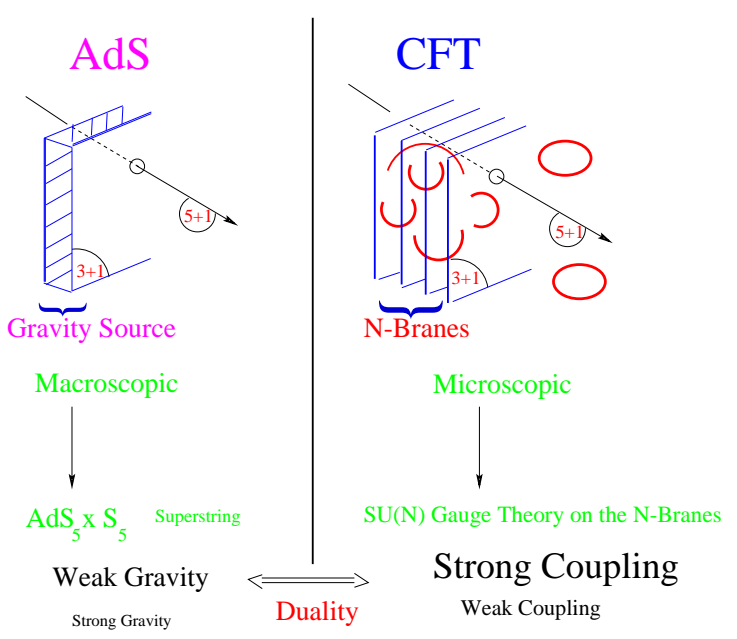

Figure 2. $\mathrm{AdS}_{5} / \mathrm{CFT}_{4}$ duality correspondence.

field theories at strong coupling and string gravity at weak coupling in the limit of large number of colours $\left(N_{c} \rightarrow \infty\right)$, see Fig.2. It can be examined quite precisely in the $\mathrm{AdS}_{5} / \mathrm{CFT}_{4}$ case which conformal field theory corresponds to $S U(N)$ gauge theory with $\mathcal{N}=4$ supersymmetries.

Some existing extensions to other gauge theories with broken conformal symmetry with less or no supersymmetries will be valuable for our approach, since they lead to confining gauge theories which are more similar to QCD. Indeed, one important question is to examine to what extent confinement plays a rôle in the Reggeization of amplitudes.

However, note that the appropriate string gravity dual of QCD has not yet been identified, and thus we are forced to restrict for the moment our use of AdS/CFT correspondence to features which are expected to be a general feature of confining theories duals.

Let us schematically recall the canonical derivation of the $\mathrm{AdS}_{5}$ background. One starts from the (super)gravity classical solution of a system of $N D_{3}$-branes in a $10-D$ space of the (type IIB) superstrings. The metrics solution of the (super)Einstein equations read

$$
d s^{2}=f^{-1 / 2}\left(-d t^{2}+\sum_{1-3} d x_{i}^{2}\right)+f^{1 / 2}\left(d r^{2}+r^{2} d \Omega_{5}\right),
$$
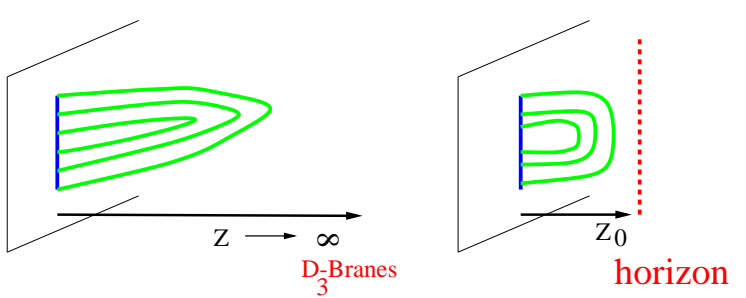

Figure 3. Exemple of minimal surfaces with Wilson lines boundary.

where the first four coordinates are on the brane and $r$ corresponds to the coordinate along the normal.

$f=1+\frac{R^{4}}{r^{4}} ; \quad R=4 \pi g_{Y M}^{2} \alpha^{\prime 2} N$,

and $g_{Y M}^{2} N$ is the 't Hooft-Yang-Mills coupling and $\alpha^{\prime}$ the string tension. Then one introduce the "Maldacena limit", where one sits near-by the branes while in the same time going to weak string coupling limit. the space-time is thus distorted due to the (super) gravitational field of the branes. One writes

$\frac{\alpha^{\prime}(\rightarrow 0)}{r(\rightarrow 0)} \rightarrow z, R$ fixed $\Rightarrow g_{Y M}^{2} N \sim \frac{1}{\alpha^{2}} \rightarrow \infty$.

By reorganizing the two parts of the metrics one obtains

$d s^{2}=\frac{1}{z^{2}}\left(-d t^{2}+\sum_{1-3} d x_{i}^{2}+d z^{2}\right)+R^{2} d \Omega_{5}$,

which corresponds to the $\mathrm{AdS}_{5} \times S_{5}$ background structure, $S_{5}$ being the 5 -sphere. More detailed analysis shows that the isometry group of the 5sphere is the geometrical dual of the $\mathcal{N}=4$ supersymmetries.

In order to illustrate the way how one formulates the AdS/CFT correspondence, let us consider the example of the vacuum expectation value (vev) of Wilson lines in a configuration parallel to the time direction of the branes. This configuration allows a determination of the potential between colour charges [2].

One writes

$\left\langle e^{i P \int_{C} \vec{A} \cdot \overrightarrow{d l}}\right\rangle=\int_{\Sigma} e^{-\frac{A r e a(\Sigma)}{\alpha^{\prime}}} \approx e^{-\frac{A r e a_{m i n}}{\alpha^{\prime}}} \times$ Fluct., 
where $C$ is the Wilson line contour near the $D_{3}$ branes and $\Sigma$ the surface in $A d S$-space with $C$ as the boundary, see Fig.3. The minimal area approximation is the vev evaluation classical $\alpha^{\prime} \rightarrow 0$ limit which can eventually be improved by calculating the fluctuation determinant around the minimal surface. In Fig. 3, we have sketched Two cases: the $A d S_{5}$ "conformal" one and a a confining case, $A d S_{B H}$, where a black-hole $(\mathrm{BH})$ in the AdS bulk determines a characteristic horizon scale $R_{0}$ breaking conformal invariance (see Witten [酉]).

The vev results can be summed up as follows:

$$
\begin{aligned}
A d S_{5}:\langle\text { Wilson Lines }\rangle & =e^{T V(L)} \sim e^{\# T / L} \\
A d S_{B H} & :\langle\text { Wilson Lines }\rangle=e^{T V(L)} \sim e^{\# T L / R_{0}^{2}}
\end{aligned}
$$

where, the potential behaviour is as expected for respectively conformal (perimeter law) and confining (area law) cases. Note that there is interesting information in the coupling dependent numbers here denoted by \# .

\section{Supergravity Duals of Scattering Am- plitudes}

Interestingly enough, high energy amplitudes in gauge field theories can be related to other related configurations of minimal surfaces [3]. At high energy, fast moving colour sources propagate along linear trajectories in coordinate space thanks to the eikonale approximation. An analytic continuation from Minkowski to Euclidean $\mathcal{R}^{4}$ space allows one to find a geometrical interpretation in terms of a well-defined minimal surface problem. Let us consider for illustration different applications.

\section{1. "Quark" elastic scattering}

Calling "(anti)quarks" the colour sources in the (anti)fundamental representation of $\mathrm{SU}(\mathrm{N})$, the high-energy elastic quark-(anti)quark amplitude can be written 沟

$A\left(s, q^{2}\right)=2 i s \int d \vec{l} e^{i \vec{q} \cdot \vec{l}}\left\langle W_{1} W_{2}\right\rangle_{L=|\vec{l}|}^{\chi=\log s / m^{2}}$,

where $\vec{l}$ is the impact parameter between the two trajectories, conjugated to the momentum trans-

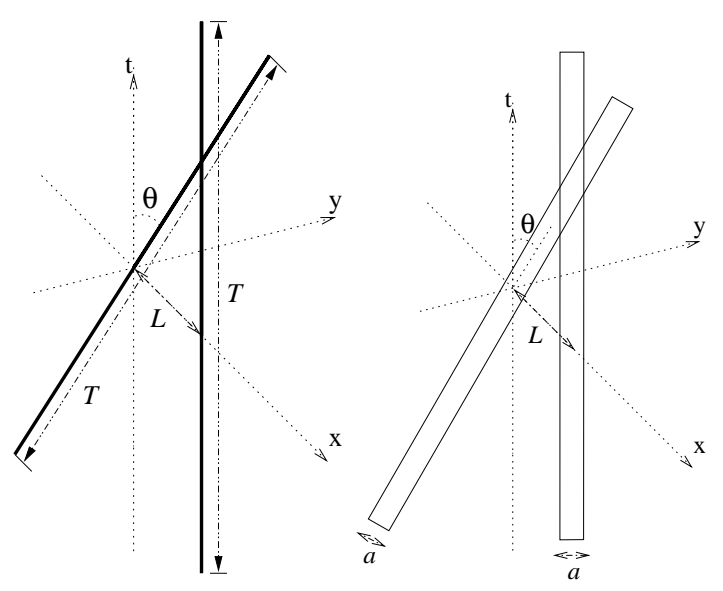

Figure 4. Wilson lines for "quark" and dipole elastic scattering in $\mathcal{R}^{4}$.

fer $\vec{q}, \chi$ the total rapidity interval. Performing an analytic continuation to the Euclidean space:

$\chi \rightarrow i \theta ; t_{\text {Mink }} \rightarrow-i t_{\text {Eucl }}$,

the Wilson line vev can be expressed as a minimal surface problem whose boundaries are two straight lines with an angle $\theta$ in $\mathcal{R}^{4}$, see Fig. 1 . In flat space, with the same boundary conditions, the minimal surface is the helicoid. One thus realizes that the problem can be formulated as a minimal surface problem whose mathematical formal solution is a generalized helicoidal manifold embedded in Euclidean AdS Spaces.

\subsection{Dipole elastic scattering}

For elastic scattering of colourless states, it is interesting to consider QCD dipoles, which are known to be a good toy model. Their propagation in coordinate space within the eikonale approximation can be represented by elongated Wilson loops near both right and left moving light-cone directions. Using the same analytic continuation framework, one has to compute the Wilson loop correlator in the configuration displayed in Fig. 4 .

The minimal area solution with the corresponding boundary conditions is difficult to find in analytic form, necessary for the continuation to Minkowski space. Some approximation schemes may be used [2]. A quite general and intringuing 


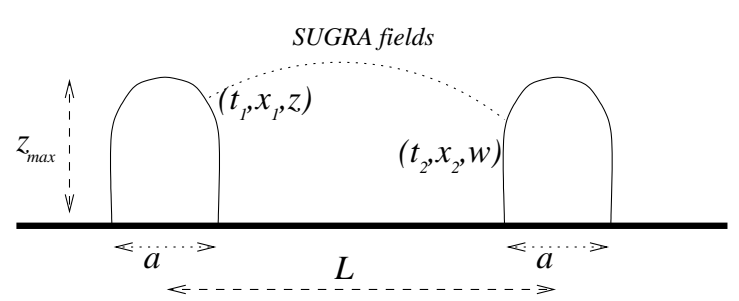

Figure 5. Dipole scattering at large impact parameter.

feature is the existence of a geometrical transition between small and large impact parameter, corresponding to the realization of disconnected minimal surfaces, see Fig 5 .

\subsection{Dipole inelastic scattering}

The application of AdS/CFT correspondence for the two previous exemples is not so easy, even if partial results are encouraging. For "quark" elastic scattering, an infra-red time-like cut-off is to be introduced due to the colour charges of the quarks which implies a regularization scheme and a complication of the geometrical aspects. For dipole elastic scattering, there is no need for a cut-off but the geometry of the minimal surface is complicated. Inelastic scattering of dipoles allows one to circumvent these difficulties. Indeed, the helicoidal geometry remains valid due to the eikonale approximation for the "spectator quarks" while the "exchanged quarks" define a trajectory drawn on the helicoid, see Fig.6. This trajectory plays the rôle of a dynamical time-like cut-off which takes part in the minimization procedure.

Adopting the "world-line" path integral scheme of Feynman [5], one may write the inelastic amplitude in terms of a Wilson loop vev:

$$
\int \mathcal{D} \tau\left\langle W\left(1 \rightarrow 3^{\prime} \rightarrow 4^{\prime} \rightarrow 2^{\prime} \rightarrow 1^{\prime}\right)\right\rangle_{\mathcal{A},[\tau]} e^{-2 m \mathcal{L}[\tau]},
$$

where $\tau$ parametrizes the boundary trajectories and $\mathcal{L}$ is their total length. Using the AdS-CFT correspondence in the same framework as previously, one may formally integrate over the gauge degrees of freedom and write

$$
\left\langle W\left(1 \rightarrow 3^{\prime} \rightarrow 4^{\prime} \rightarrow 2^{\prime} \rightarrow 1^{\prime}\right)\right\rangle_{\mathcal{A},[\tau]}=e^{-\frac{A r e a[\tau]}{2 \pi \alpha^{\prime}}} \times \text { Fluct }
$$
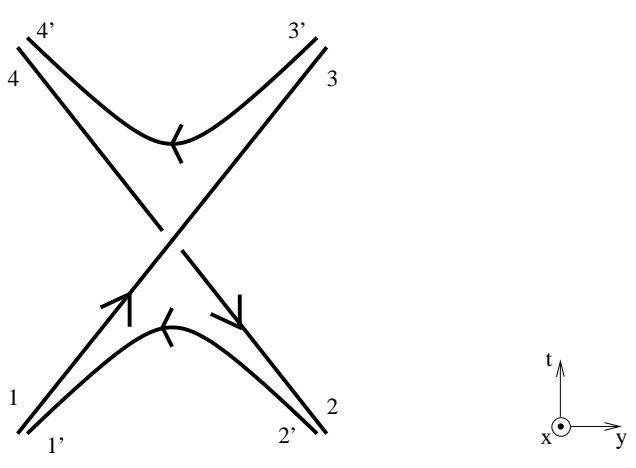

Figure 6. Wilson lines for inelastic dipole scattering

Note that the remaining minimization in $\tau$ runs now on both the area and its boundary.

\section{Reggeization from the geometry of its AdS dual}

To give a practical exemple of calculation we focus on the configuration of Wilson lines of Fig.6 in the context of a confining theory, dual to the $A d S_{B H}$ case with a limiting horizon, see Fig. 3 . The $\operatorname{AdS}_{B H}$ metrics, as well as the canonical $\mathrm{AdS}_{5}$ one, are characterized by a singularity at $z=0$ which implies a rapid growth in the $z$ direction towards the $\mathrm{D}_{3}$ branes, then stopped near the horizon at $z_{0}$. Thus, to a good approximation, and for large enough impact parameter (compared to the horizon distance), the main contribution to the minimal area is from the metrics in the bulk near $z_{0}$ which is nearly flat. Hence, near $z_{0}$, the relevant minimal area can be drawn on a classical helicoid . Parametrizing the helicoid:

$$
\begin{aligned}
t & =\tau \cos \theta \sigma / L \\
y & =\tau \sin \theta \sigma / L \\
x & =\sigma \\
z & \sim z_{0}
\end{aligned}
$$

the solution of the amplitude boils down to an Euler-Lagrange minimization over $\tau$, namely

$$
\begin{aligned}
A_{\mathcal{R}}\left(s, L^{2}\right) & \propto \frac{1}{s} \lim _{\alpha^{\prime} \rightarrow 0} \int \mathcal{D} \tau e^{-\frac{1}{2 \pi \alpha^{\prime}} \operatorname{Area}[\tau]} \times \\
& \times e^{-2 m \mathcal{L}[\tau]} \times \text { Fluct. }
\end{aligned}
$$




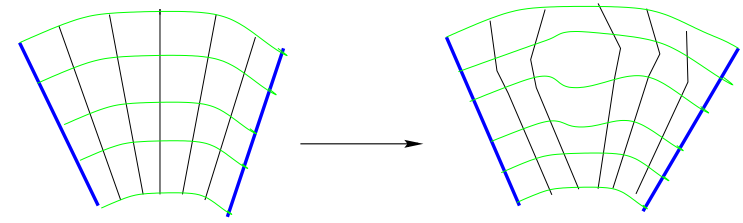

Figure 7. Fluctuations around the minimal surface

where Area $[\tau]$ is the section of an helicoid bounded by the quark trajectories having total length $\mathcal{L}[\tau]$. Note that the kinematical factor $1 / s$ in front comes from an infinite-dimensional fermionic spin factor along the quark trajectories corresponding to a bosonized representation 5. Its non-trivial calculation makes use of the 3dimensional embedding of the quark trajectories on the helicoid surface [2].

After some technical steps and analytic continuation back to Minkowski space, the resulting amplitude reads:

$A_{\mathcal{R}}\left(s, q^{2}\right)=\int d \vec{l} e^{i \vec{q} \cdot \vec{l}} e^{-\frac{L^{2}}{4 \alpha_{e f f}^{\prime} \chi}} \propto s^{-\alpha_{e f f}^{\prime} q^{2}}$,

corresponding to a linear Regge trajectory with intercept 0 and slope $\alpha_{\text {eff }}^{\prime}$ related to the quark potential calculated within the same AdS/CFT framework.

A semi-classical correction comes from the fluctuations near the minimal surface sketched in Fig.7. It can be shown to be intimately related to a contribution to the quark potential similar to the well-known Lüscher term [6]. One finally finds

$A_{\mathcal{R}}\left(s, q^{2}\right) \propto s^{\frac{n_{1}}{24}-\alpha_{e f f}^{\prime} q^{2}}$,

where $n_{\perp}$ is the number of transverse zero-modes (zero-mass transverse excitations of the string) in the AdS dual theory. It gives a shift in the intercept of the reggeon trajectory.

The value of this intercept depends on the particular realization of the AdS/CFT duality. In known examples (see [2]) it is quoted to be $n_{\perp}=7$ or 8 , , to be compared with the ordinary Lüscher term having $n_{\perp}=2$.. Note that fermionic d.o.f. are not expected to remain mass- less in an AdS background, and thus to give contributions with opposite sign.

It is worth comparing the resulting inelastic dipole amplitude with the one obtained for dipole elastic scattering in the same framework. With approximations required by to the non-trivial geometry of dipole loops one finds:

$A_{\mathcal{P}}\left(s, q^{2}\right) \sim s^{1+\frac{n_{\perp}}{96}-\frac{\alpha_{e f f}^{\prime}}{4} q^{2}}$,

with the same values of $\alpha_{e f f}^{\prime}$ and $n_{\perp}$. Reggeon and Pomeron Regge trajectories are thus linear and related to the quark potential. Note the factor $1 / 4$ in the exponent of $A_{\mathcal{P}}$ with respect to $A_{\mathcal{R}}$ which has some consistency with actual amplitudes, since it gives phenomenologically consistent Pomeron and (average) dominant Reggeon trajectories [2].

\section{Conclusion: Reggeization and confine- ment}

Lattice calculations, which is the only presently known way to evaluate directly QCD observables at strong coupling, are not able to compute highenergy amplitudes. hence, an interesting output of the application of AdS/CFT correspondence to high energy amplitudes at strong coupling is to discuss the relation between Reggeization and confinement, using the description in the dual theory.

When comparing $\mathrm{AdS}_{5}$ duality - which corresponds to a conformal, non-confining gauge theory - with $\mathrm{AdS}_{B H}$ duality, which leads to reggeization, the difference ultimately comes from the different metrics in the bulk and hence from the minimal surfaces for the same boudary conditions. Taking into account their different geometry, see e.g. Fig.3, one expects after analytic continuation and in the large energy $(\chi \rightarrow \infty)$ limit:

$\operatorname{Area}_{\min }^{A d S} \sim \lim _{\chi \rightarrow \infty} \frac{L}{L / \chi} ;$ Area $_{\min }^{B H} \sim \lim _{\chi \rightarrow \infty} L \times \frac{L}{\chi}$.

The $\mathrm{AdS}_{5}$ case leads to a $L$-invariant value and, after Fourier transformation, to a high-energy amplitude with a $q^{2}$ independent energy exponent (or flat Regge trajectory). On the other hand, the 
$\mathrm{AdS}_{B H}$ case leads to a linear Regge trajectory after Fourier transformation. For the $\mathrm{AdS}_{B H}$ case this rough expectation can be verified by an explicit calculation. Hence confinement appears as an essential ingredient for the reggeized structure of high-energy amplitudes. We expect this result not to be dependent on the precise geometrical $\mathrm{AdS}_{B H}$ setting and thus to indicate a quite general property of confining theories.

As a conclusion, let us discuss the list of problems for which the AdS/CFT framework give new insights on the 35-years-old puzzle of high-energy amplitudes at strong gauge coupling 1?. As usual this also adds some new problems in this context! Among the new insights:

- String dimensionality: $D=4+1+5$ : Extradimensions play an important dynamical rôle.

- Gravitation: It is decoupled. The (classical) Pomeron intercept is $1+\epsilon$ instead of 2 for the graviton. $\epsilon$ is related to a Lüsher term.

- Regge trajectories: They come out linear, with slopes and intercepts related to the quark potential including a Lüscher term.

Among the problems:

- High-energy phenomenology: Many aspects, like the Flavor/Spin dependences, remain to be studied.

- Approximations: The dual gauge theory is not specified, and the exact minimal surface in the bulk metrics to be determined.

- Dual of $Q C D$ ? In the present framework, the confining scale $R_{0}$ has no relation with $\Lambda_{Q C D}$.

- Unitarity: A more complete investigation requires the study of multi-leg amplitudes.

- Deeper general problems: The formulation of string theory in AdS backgrounds and last but not least, a proof of the AdS/CFT conjecture.

\section{Acknowledgement}

The whole approach of high-energy amplitudes described in this review paper comes from a collaboration with Romuald Janik from Cracow University.

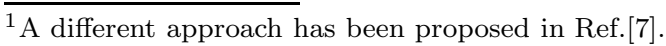

\section{REFERENCES}

1. J. Maldacena, Adv. Theor. Math. Phys. 2 (1998) 231; S.S. Gubser, I.R. Klebanov and A.M. Polyakov, Phys. Lett. B428 (1998) 105; E. Witten, Adv. Theor. Math. Phys. 2 (1998) 253; , Adv. Theor. Math. Phys. 2 (1998) 505. O. Aharony, S.S. Gubser, J. Maldacena, H. Ooguri and Y. Oz, Phys.Rept. 323 (2000)183.

2. J. Maldacena, Phys. Rev. Lett. 80 (1998) 4859; S.-J. Rey and J. Yee, hep-th/9803001; JJ. Sonnenschein and A. Loewy, JHEP 0001 (2000) 042. Y. Kinar, E. Schreiber, J. Sonnenschein and N. Weiss, Nucl. Phys. B583 (2000) 76.

3. R.A. Janik and R. Peschanski, Nucl. Phys. B586 (2000) 163 , Nucl. Phys. B565 (2000) 193 , Nucl. Phys. B565 (2000) 193; R.A. Janik, Phys. Lett. B500 (2001) 118. See also: M. Rho, S.-J. Sin and I. Zahed, Phys. Lett. B466 (1999) 199.

4. O. Nachtmann, hep-ph/9609365. E. Meggiolaro, Z. Phys. C76 (1997) 523.

5. R.P. Feynman, Phys. Rev. 80 (1950) 440 A. M. Polyakov, Mod. Phys. Lett. A3 (1988) 325. G. P. Korchemsky, Int. J. Mod. Phys. A7 (1992) 339; , Phys. Lett. B232 (1989) 334.

6. M. Lüscher, K. Symanzik and P. Weisz, Nucl.Phys. B173 (1980) 365. O. Alvarez, Phys.Rev. D24 (1981) 440.

7. J. Polchinski and M.J. Strassler, Phys. Rev. Lett. 88 (2002) 031601; , hep-th/0209211. 